\title{
Giving Attention to Generative VAE Models for de novo Molecular Design
}

\author{
Orion Dollar ${ }^{1}$, Nisarg Joshi ${ }^{1}$, David A.C. Beck ${ }^{1,2^{*}}$, Jim Pfaendtner ${ }^{1 *}$ \\ ${ }^{1}$ Department of Chemical Engineering, University of Washington, Seattle 98185, WA, USA; \\ ${ }^{2}$ eScience Institute, University of Washington, Seattle 98185, WA, USA. \\ *Email: odollar@uw.edu, jpfaendt@uw.edu, dacb@uw.edu
}

\begin{abstract}
We explore the impact of adding attention to generative VAE models for molecular design. Four model types are compared: a simple recurrent VAE (RNN), a recurrent VAE with an added attention layer (RNNAttn), a transformer VAE (TransVAE) and the previous state-of-the-art (MosesVAE). The models are assessed based on their effect on the organization of the latent space (i.e. latent memory) and their ability to generate samples that are valid and novel. Additionally, the Shannon information entropy is used to measure the complexity of the latent memory in an information bottleneck theoretical framework and we define a novel metric to assess the extent to which models explore chemical phase space. All three models are trained on millions of molecules from either the ZINC or PubChem datasets. We find that both RNNAttn and TransVAE models perform substantially better when tasked with accurately reconstructing input SMILES strings than the MosesVAE or RNN models, particularly for larger molecules up to 700 Da. The TransVAE learns a complex "molecular grammar" that includes detailed molecular substructures and high-level structural and atomic relationships. The RNNAttn models learn the most efficient compression of the input data while still maintaining good performance. The complexity of the compressed representation learned by each model type increases in the order of MosesVAE $<$ RNNAttn $<\mathrm{RNN}<$ TransVAE. We find that there is an unavoidable tradeoff between model exploration and validity that is a function of the complexity of the latent memory. However, novel sampling schemes may be used that optimize this tradeoff and allow us to utilize the information-dense representations learned by the transformer in spite of their complexity.
\end{abstract}




\section{Introduction}

The design and optimization of molecular structures for a desired functional property has the potential to be greatly accelerated by the integration of deep learning paradigms within existing scientific frameworks for molecular discovery. Traditional "direct" design approaches, in which a set of molecules are selected based on expert intuition and tested for a given property, are often time-consuming and require extensive resources to explore a small, local region of chemical phase space. ${ }^{1}$ By contrast, "inverse" approaches, in which structures are derived based on their likelihood to exhibit a given property value, are desirable as they are far less limited in scope and allow for highthroughput screening of thousands to hundreds of thousands of structures. ${ }^{2}$ Given the size and complexity of chemical phase space, ${ }^{3}$ successful implementation of an inverse design algorithm would allow researchers to reach global structural optima more rapidly thereby increasing the speed of discovery.

A variety of deep generative model architectures have been explored for this purpose ${ }^{4}$, with a particular focus given to the variational autoencoder (VAE) ${ }^{5-10} \mathrm{~A}$ VAE is capable of broadcasting a machine-interpretable representation of molecular structure (e.g. a SMILES string ${ }^{11}$, SELFIES string ${ }^{12}$ or molecular graph $^{13}$ ) to a dense, continuous latent space or "model memory". This memory has several unique features that make VAEs promising for inverse design: (i) It can be embedded with a property and thus serve as an approximation of the joint probability distribution of molecular structure and chemical property. (ii) During training, it will organize itself meaningfully so that similar molecules are near each other in phase space. (iii) Due to its mapping from discrete to continuous data, it can be navigated with gradient-based optimization methods. ${ }^{14}$

In spite of these benefits, generative VAE models suffer from a set of complicating issues that have been the focus of much recent work. Although more robust than their adversarial counterparts, VAEs are still subject to experiencing posterior collapse in which the decoder learns to ignore the latent memory altogether and reconstruct a fuzzy approximation of the input distribution. ${ }^{15}$ On the other hand, even with a meaningful posterior there are often pockets of phase space within the latent memory that do not map to any valid chemical structures. Many recent innovations in architecture, featurization and hyperparameter selection have centered around these problems and have proven quite successful at improving reconstruction accuracy and sampling validity. ${ }^{13,16,17}$

However, we lack a holistic view of the effect of these improvements on the practical utility of a model's latent memory. For instance, metrics to examine the diversity and novelty of sampled molecules are not well-defined. ${ }^{18}$ These traits are arguably as important as validity, if not more so. Generating samples is orders of magnitude faster than training and a model that can generalize to regions of chemical phase space far outside the training set is extremely valuable for exploration. Although fewer studies have evaluated generative VAE models in this way, the results reported in the Moses Benchmarking Platform indicate that there is still significant room for improvement. ${ }^{19}$ 
The rapid technological progression within the field of natural language processing (NLP) may offer some hints towards a future where AI-designed molecules are the norm rather than the exception. Despite the overwhelming number of similarities between model architectures used for molecular generation and those used for NLP, the stateof-the-art in the former lags notably behind that of the latter. Attention mechanisms, which have produced some of the more impressive feats in AI in recent memory, have been used sparingly for graph-based analysis of chemical structure $^{20}$ and have not yet been incorporated into any generative algorithms. Attention-based models can learn longrange syntactical dependencies within a sequence and have shown immense capabilities in machine translation ${ }^{21}$, text generation $^{22}$, protein structure prediction ${ }^{23}$ and AI-composed music ${ }^{24}$. They've also shown a surprising aptitude for style with their ability to combine wit, poetic prose and the tenets of philosophy into cogent metaphysical selfreflections on the meaning of virtual existence ${ }^{25,26}$. Although perhaps not as amusing, we anticipate they may exhibit a similar sense of coherence when tasked with exploring novel chemistries.

An examination of the performance of standard recurrent neural networks (RNN), RNN+Attention and Transformer VAE architectures for the purpose of molecular generation follows. We show the effect of attention on reconstruction accuracy for both the ZINC and PubChem datasets. Novel metrics are proposed that define the models' ability to explore new regions of chemical phase space and compare the relative information density of the latent memory. We show that for all model types there exists a relationship between sample validity and exploration that mimics closely the tradeoff between complexity and generalization within an information bottleneck. Finally, we suggest a simple sampling scheme that offers a compromise between the two and look towards a future where we may optimize this directly during training with more precise control during the nascent development of the latent memory.

\section{Variational Autoencoder and the Information Bottleneck}

A VAE consists of an encoder that takes a sequence as input, i.e., a SMILES string, and a decoder that attempts to reconstruct the input as accurately as possible. ${ }^{27}$ Prior to decoding, the encoder transforms the input, $\boldsymbol{x}$, into an intermediate latent representation, $\boldsymbol{z}$, that serves as the "model memory." Information is bottlenecked between the encoder and decoder such that $d_{\text {latent }} \ll d_{\text {input }}$ where $d$ is the dimensionality of a given layer. In this sense a VAE can be thought of as a compression algorithm that produces compact, information dense representations of molecular structures. The encoder learns how to compress the input data and the decoder learns how to reconstruct the full sequence from the compressed representation (Fig. 1).

The training objective seeks to minimize the reconstruction loss between the input and output while simultaneously learning the ground truth probability distribution of the training data. The latter half of this objective is especially important to the generative capacity of the model. Knowledge of the marginal likelihood, $p(\boldsymbol{x} \mid \boldsymbol{z})$, allows us to 


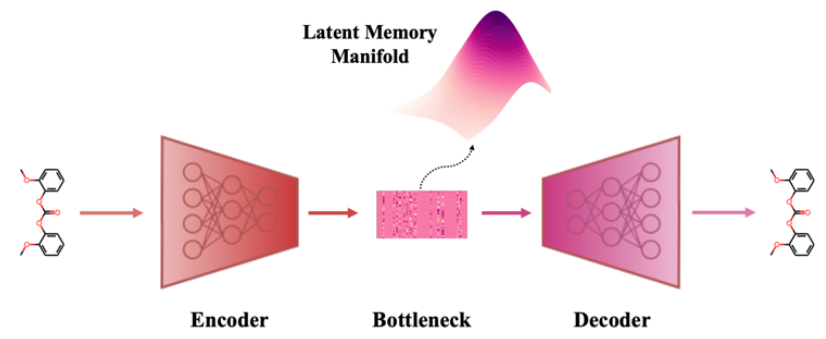

Figure 1. Major structural components of the VAE architecture. A machine-interpretable representation of a molecular structure is sent to an encoder where it is compressed to a dense latent representation within the bottleneck. Each of the compressed molecular embeddings represent one point within a larger probability manifold aka "model memory". During training, the model learns to fit this manifold to the true probability distribution of the input data. To ensure the compressed embeddings contain structurally meaningful information, they are sent to a decoder which learns to reconstruct the original molecular structure.

directly sample new data points by first querying from the model's memory, $z$, and then decoding. To achieve this, we assume the true posterior can be adequately approximated by a set of Gaussians. The Kullbach-Leibler divergence $(\mathrm{KLD})^{28}$ between $\boldsymbol{z}$ and the standard normal distribution $\mathcal{N}(0,1)$ is minimized alongside the reconstruction loss and thus the full objective function can be formalized according to the variational lower bound as

$$
\log p_{\theta}(\boldsymbol{x} \mid \mathbf{z}) \geq \mathcal{L}(\theta, \phi ; \boldsymbol{x}, \boldsymbol{z})=\mathbb{E}_{q_{\phi}(\mathbf{z} \mid \boldsymbol{x})}\left[\log p_{\theta}(\boldsymbol{x} \mid \mathbf{z})\right]-D_{K L}\left(q_{\phi}(\mathbf{z} \mid \boldsymbol{x})|| p(\mathbf{z})\right)
$$

where the term on the left is the reconstruction loss of the decoder, $p_{\theta}(\boldsymbol{x} \mid \mathbf{z})$, and the term on the right is the KLD loss between the encoder output, $q_{\phi}(\mathbf{z} \mid \boldsymbol{x})$, and the standard normal distribution, $p(\mathbf{z})$.

Intuitively, the addition of gaussian noise can be thought of as a way to increase the "spread" of samples within the latent memory. Rather than encoding individual molecular structures as a single point in phase space, it encodes them as a probability distribution. This allows the model to smoothly interpolate between the continuous representations of known molecular structures and make informed inferences outside of the set of training samples.

The latent memory can also be analyzed within the framework of information bottleneck (IB) theory. ${ }^{29}$ During compression, there is an unavoidable tradeoff between the amount of useful information stored in the model's memory and the amount of low information complexity stored in the model's memory. ${ }^{30}$ The IB objective can be written as

$$
\max _{\theta, \phi}\left[I\left(q_{\phi}(\mathbf{z} \mid \boldsymbol{x}) ; p_{\theta}(\boldsymbol{x} \mid \mathbf{z})\right)-\beta I\left(\boldsymbol{x} ; q_{\phi}(\mathbf{z} \mid \boldsymbol{x})\right)\right]^{31}
$$

where $I$ is the mutual information between two variables and $\beta$ is a scaling parameter. We seek a solution that maximizes the amount of mutual information between the latent memory and the reconstructed output while minimizing the amount of mutual information between the latent memory and the input. Since there is rarely a unique solution to the reconstruction objective, the $\beta$ parameter discourages the model from finding a needlessly complex (but still valid) local minimum. Thus, in addition to controlling the "spread" of information, the KLD term 
can be interpreted as a filter of irrelevant information with pore size $1 / \beta$. It will be useful to keep this framework in mind as we observe the development of the latent memory during training.

\section{Adding Attention to the VAE}

The encoder and decoder of a VAE are implemented as two sets of neural network hidden layers. Current best practice on generative VAEs for molecular design calls for both the encoder and decoder to be built from gated recurrent unit (GRU) layers, ${ }^{19}$ however convolutional layers were originally thought to be better suited for the encoder and have also been used in some studies. ${ }^{14,32}$ Due to the simplicity and generality of the encoder-decoder style of model, it is feasible to build VAEs in a modular fashion; swapping out different encoder and decoder architectures while maintaining the variational restrictions of the bottleneck between them.

a)

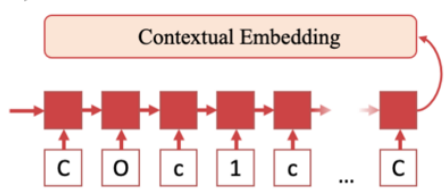

b)

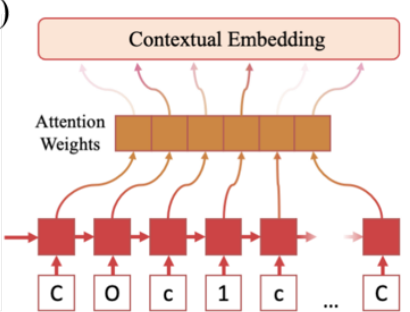

c)

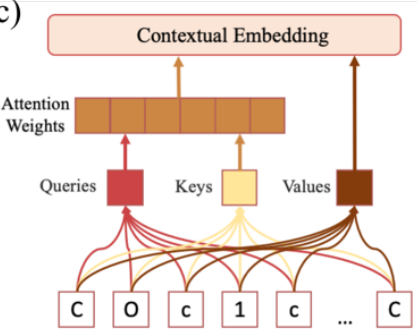

d)

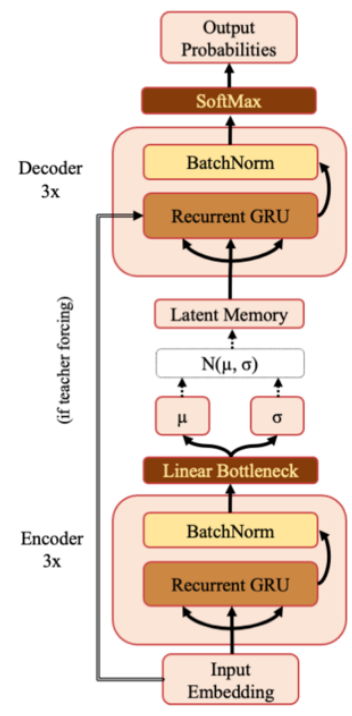

RNN

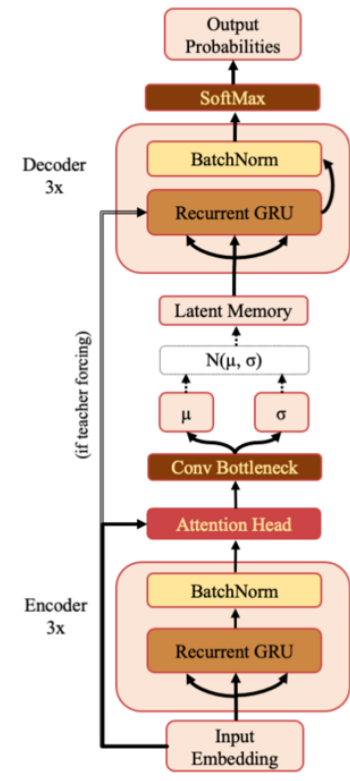

RNNAttn

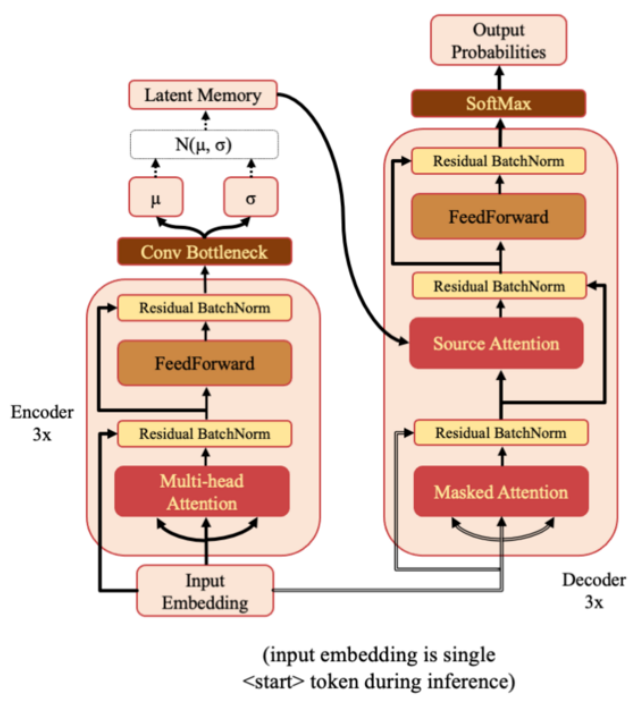

Transformer

Figure 2. a-c) Schematic illustrations of the sequential layers for each model type - RNN (a), RNNAttn (b) and Transformer (c). d) Full schematics for each model type. The RNN model consists of three recurrent GRU layers in both the encoder and decoder. The RNNAttn model has the same architecture as the RNN with the addition of a single attention head after the final recurrent GRU layer in the encoder. The transformer is modeled after the original implementation as reported by Viswani et al ${ }^{55}$ However, rather than passing the output of the encoder directly into the source attention layer, the encoder output is first stochastically compressed and then fed into the decoder. 
In standard RNNs, the first recurrent cell takes the first element of the sequence and outputs a hidden state. That hidden state is then propagated down the sequence with each subsequent recurrent cell taking the previous cell's hidden output and the next sequence element as inputs until the entire sequence has been traversed. The final hidden state is the "contextual embedding" of the sequence (Fig. 2a). In some architectures the contextual embedding and the latent memory may be the same size. However, oftentimes there will be an additional set of linear bottleneck layers that further compress the output of the encoder GRU layers $\left(d_{\text {encoder }} \rightarrow d_{\text {latent }}\right)$.

In attention-based recurrent models (RNNAttn), the flow of information proceeds similarly to a standard RNN. However rather than only using the final hidden output state, a weighted combination of all the hidden states along the sequence is used as the contextual embedding (Fig. 2b). The attention weights are learned during training by letting the input sequence "attend" to its own hidden state matrix. This allows the model to eschew the linearity imposed by the RNN architecture and learn long-range dependencies between sequence elements.

Transformer (Trans) models remove recurrence altogether and exclusively use attention head layers. ${ }^{33}$ The inputs are a set of keys, values and queries transformed from the initial input sequence that are sent through a series of matrix multiplications to calculate the attention weights and the contextual embedding (Fig. 2c). The set of values are analogous to the hidden state matrix output of an RNN and the attention weights are determined by matrix multiplication of the keys and queries. Transformers have the advantage of reducing the path length of information traveling through the model and are highly parallelizable.

As the size of the contextual embedding is significantly larger for the two attention-based architectures vs. the simple recurrent architecture $\left(n_{\text {seq }} x d_{\text {encoder }} v s\right.$. $\left.d_{\text {encoder }}\right)$, using a linear bottleneck is practically infeasible because it would explode the number of trainable parameters. Instead, a convolutional bottleneck similar to those used in generative image nets ${ }^{34}$ is employed.

Table 1. Model Architectures. The dimensionality of the model ( $\left.d_{\text {model }}\right)$ is defined as the size of the sequential layers. Recurrent model names are written as ModelType- $\left\{d_{\text {model }}\right\}$. Transformer model names are written as Trans $\left\{d_{f e e d f o r w a r d} / d_{\text {model }}\right\} x-\left\{d_{\text {model }}\right\}$. All models used in this study have a latent dimensionality of size 128.

\begin{tabular}{cccc} 
Model Type & $\boldsymbol{d}_{\text {model }}$ & $\boldsymbol{d}_{\text {latent }}$ & $\boldsymbol{d}_{\text {feedfoward }}$ \\
\hline RNN-128 & 128 & 128 & $\mathrm{n} / \mathrm{a}$ \\
RNN-256 & 256 & 128 & $\mathrm{n} / \mathrm{a}$ \\
RNNAttn-128 & 128 & 128 & $\mathrm{n} / \mathrm{a}$ \\
RNNAttn-256 & 256 & 128 & $\mathrm{n} / \mathrm{a}$ \\
Trans1x-128 & 128 & 128 & 128 \\
Trans4x-128 & 128 & 128 & 512 \\
Trans1x-256 & 256 & 128 & 256 \\
Trans4x-256 & 256 & 128 & 1024
\end{tabular}


The concepts of attention and the variational bottleneck have rarely been used in tandem. Of those studies that have surveyed this type of model, all have used natural language tasks as the basis of their evaluations. A variational attention-mechanism was used for sequence-to-sequence models $^{35}$ and a few novel variational transformer architectures have recently been proposed. ${ }^{36-38} \mathrm{We}$ opt for simplicity, adapting the architecture from Vaswani et al. ${ }^{33}$ with as few modifications as possible. This allows us to easily compare the bottlenecks of different model types and is sufficient for the task given the much smaller vocabulary size of SMILES strings compared to NLP vocabularies. ${ }^{39}$ Full schematics for each model type are shown in Fig. $2 \mathrm{~d}$ and model dimensions listed in Table 1. In addition to the model types listed above, we also trained the Moses implementation of a SMILES-based VAE with the hyperparameters suggested by Polykovskiy et al. ${ }^{19}$ Trained model checkpoint files and code for training models and generating samples is available at https://github.com/oriondollar/TransVAE.

\section{Results \& Discussion}

\section{Impact of Attention}

We first analyze the models' ability to reconstruct molecules from the ZINC and PubChem datasets to determine the role attention plays in learning molecular structure. One of the original motivations for the use of attention was to increase the length of sentences that could be accurately translated by machine translation models. ${ }^{21}$ Thus, we expect a similar increase in accuracy when encoding and decoding longer SMILES strings.

a)

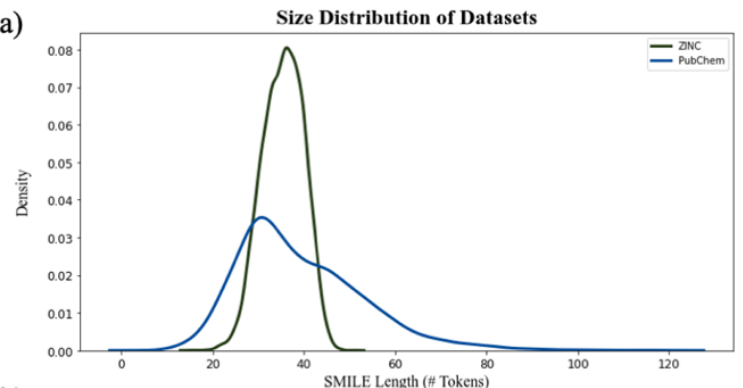

b)

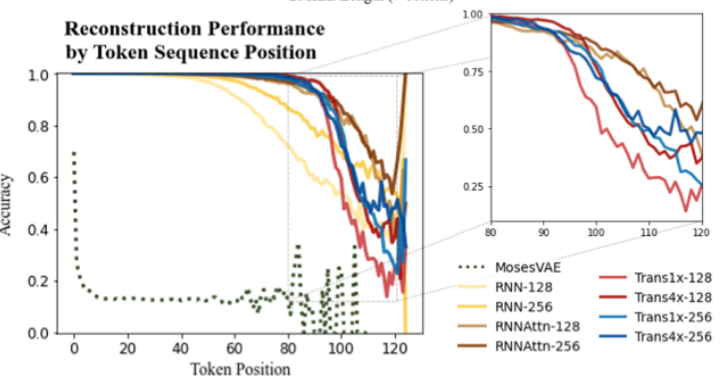

Figure 3. Assessing model reconstruction performance on the PubChem dataset (trained for 60 epochs). Input data molecular size distributions (a) and reconstruction accuracies for all model types as a function of the token position (b). Zoomed comparison of attention-based models (inset) 
Fig. 3a shows the distribution of SMILES string lengths for both datasets where length is determined by the number of tokens (excluding padding, start and stop tokens). The length of a SMILES string is highly correlated with its molecular weight (Fig. S3) and can be used as a proxy for molecular size. It is clear that by this metric the PubChem dataset has a broader distribution of sizes than ZINC. Both have approximately equal mean lengths (35.4 tokens for ZINC vs. 39.8 tokens for PubChem) however the PubChem data is significantly right skewed with a maximum token length over 50 tokens longer than the maximum within the ZINC dataset.

We can see the downstream effect that widening the molecular size distribution has on reconstruction accuracy in Fig. $3 \mathrm{~b}$ where we show the average reconstruction accuracy for all tokens at a given position within the sequence. With the exception of the Moses architecture, all of the models exhibit high fidelity reconstruction on the ZINC dataset, regardless of model type or model size (Fig. S3/Table S2). However, accuracy decreases when larger molecules are embedded into the latent memory. The model types with attention mechanisms maintain high reconstruction accuracy at longer sequence lengths than the simple recurrent models with the Trans4x-128 architecture maintaining $>99 \%$ accuracy on SMILES up to 82 tokens long ( $700 \mathrm{Da})$. This validates our hypothesis that attention will expand the number of potential applications for which these models can be used by increasing the maximum molecule size that can be reliably embedded within the latent memory.

A comparison of the two attention-based architectures (Fig. 3b inset) shows that transformers and recurrent attention models perform approximately the same until they approach the data-sparse regime of SMILES longer than $\sim 90$ tokens. At this point there is an abrupt drop in performance for the transformer models vs. a gradual decline for the recurrent attention models. The transformer appears to be more sensitive to the choice of model size as increasing the dimensionality of either its attention layers or feedforward layers improves accuracy whereas there is little performance boost when increasing the dimensionality of the recurrent attention model. Even with these improvements, the best performing transformer still exhibits a steeper decline than the worst performing recurrent attention model suggesting that a simpler attention scheme is beneficial to the model's ability to generalize on data that is outside the distribution of the training set. 
Transformer

Head 1

a)

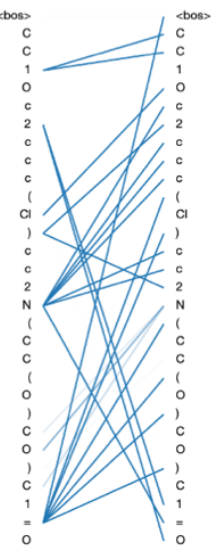

b)

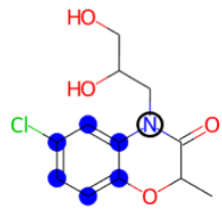

Head 2
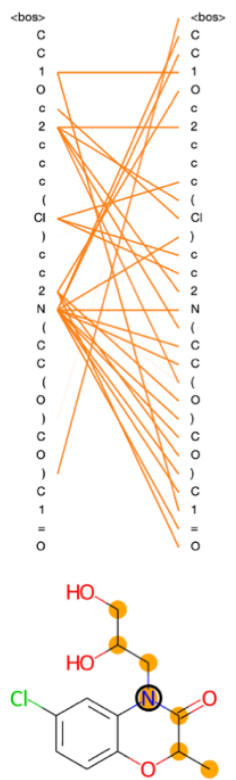

Head 3
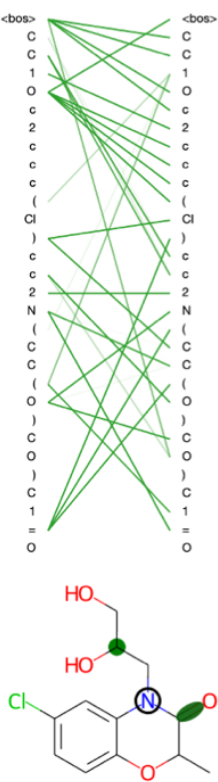

Head 4
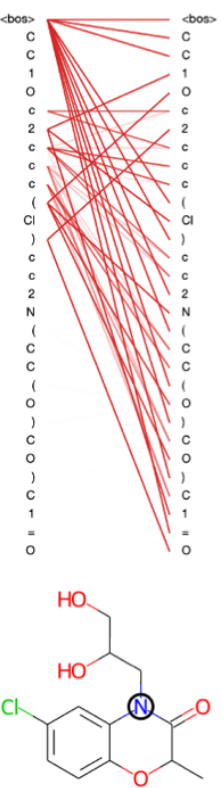

RNN+Attention
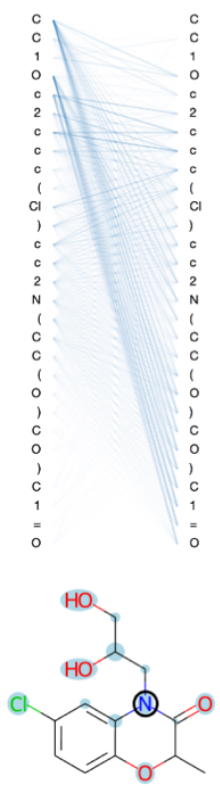

Figure 4. Analysis of the attention weights of the Trans4x-256 and RNNAttn-256 models when attending to the molecular structure of diproxadol. The full nxn set of weights are plotted for each attention head (a) using the tensor2tensor library. ${ }^{55}$ The lines show how each atom/structural feature within the SMILES string is attending to all other features within the same SMILES string (self-attention). The different patterns that emerge from each head represent a unique set of grammatical rules that the model has learned. We also show the attention of a single $N$ atom within diproxadol (b). This molecule was chosen because it is a representative example of the emergent aggregate grammatical trends. From the perspective of the nitrogen, the transformer model has identified the importance of a nearby aromatic ring (head 1), an aliphatic carbon chain of which the nitrogen is a part of (head 2) and a set of structural features including a carbon branch point and nearby double bond (head 3). The attention of the nitrogen in the RNNAttn-256 model is less focused.

There are benefits to the added complexity of the transformer, however. Analysis of the transformer attention weights reveals the model has learned a distinct set of human interpretable structural features that are much more detailed than those learned by the recurrent model with only a single attention head. We use a drug-like molecule from the ZINC dataset, diproxadol, as an illustrative example of the differences between the two (Fig. 4). The four transformer attention heads exhibit unique syntactical patterns that demonstrate the model's ability to develop its own "molecular grammar," i.e., rules that define the relationships between atoms and other structural features within a molecule including branches, double bonds, etc. Conversely, the grammar of the recurrent attention model appears to be less well-defined.

The lone nitrogen atom in diproxadol shows us how a single, centralized atom attends to its immediate molecular environment (Fig. 4b). With no supervision, the model extracts its own set of substructures that it has identified as important in relation to the nitrogen atom. Not only does it recognize defining features like the aromatic ring, it can also find non-contiguous features that depend on the structural context around a given atom (see Transformer Head 3 in Fig. 4). In this way, the machine-learned substructures are more powerful than graph-based methods that rely on a set of pre-defined substructures because they can extract contextual patterns that are difficult to pre-define but still relevant and interpretable. 
When analyzing the attention weights across a set of 5000 randomly selected molecules, we find that each attention head corresponds to a different set of higher-level relationships between atomic or structural groups such as aromatic carbons, heteroatoms, branches and rings. We assess this quantitatively by averaging the attention weights between these groups for each head (Fig. S6). As an example, the average attention weights between heteroatoms and aromatic carbons are 0.15 and 0.07 for heads 1 and 2. Conversely, the average attention weights between heteroatoms and nonaromatic carbons are $\sim 0.00$ and 0.14 for heads 1 and 2 , thus the model has partitioned information on the higher-level relationship between heteroatoms and carbon substructures based on their aromaticity. We see this directly reflected in the substructures that were extracted from the diproxadol example and show the learned weights for a variety of structures in Fig. S7. Attention plays a significant role in the machine-learned "understanding" of molecular structure and as complexity is scaled up, the extracted features become more refined and meaningful. The question then becomes how we can balance the richness of the structural features learned by the transformer with the increased complexity that is required to obtain them.

\section{Information Entropy of Model Memory}

The concept of model complexity has been alluded to, previously, as it relates to the number of attention heads, but we must also define it quantitatively. The most intuitive way to do so is to return to the framework of the information bottleneck. The latent memory provides us a uniform comparison between model types as every molecular embedding within a model's memory is the same size. By evaluating the loss function as written in Eq (2), we have instructed the model to store as much structurally relevant information within the memory as possible while also minimizing the amount of low information complexity. Therefore, we know that the complexity we have introduced qualitatively will be stored within the memory as long as that complexity contains structurally significant information. We can use the average Shannon information entropy ${ }^{40}$ across all molecular embeddings to compare the information density of latent memories between model types. This gives us a quantitative metric where a higher entropy indicates a higher degree of complexity. Others have drawn similar analogies between Shannon's entropy and system complexity, ${ }^{41}$ but to our knowledge this is the first time this metric has been introduced in the context of de novo molecular design.

To illustrate model entropy visually, we show three archetypal memory structures that we have observed in Fig. 5a. From left to right the average entropy of these memories increases from 0 nats to 127.4 nats to 393.4 nats respectively. The entropy of posterior collapse is zero because it has learned the same embedding regardless of the input molecule thus the decoder does not receive new information from the memory. The selective structure is the most commonly observed (Fig. S8) and occurs when the dimensionality of the true probability manifold is smaller than the number of latent dimensions given to the model. ${ }^{34}$ In this case the model learns to ignore superfluous dimensions, assigning them a mean of zero and standard deviation of 1 to satisfy the KLD loss requirement. We consider the other dimensions meaningful because they contribute to the total information entropy of the memory. The smeared structure is an interesting case in which the burden of information is shared across all dimensions but with each contributing less 
entropy than the meaningful dimensions from the selective structure. The smeared structure appears as a sudden phase change during training when the number of meaningful dimensions approaches zero (Fig. 5b). This effect was only observed for the MosesVAE model.

a)
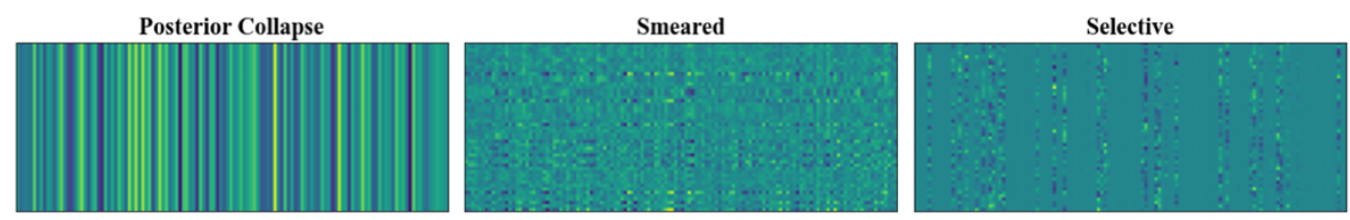

b)

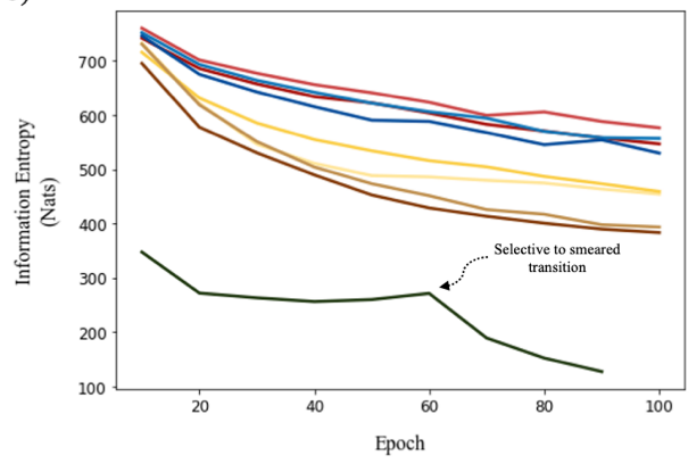

c)
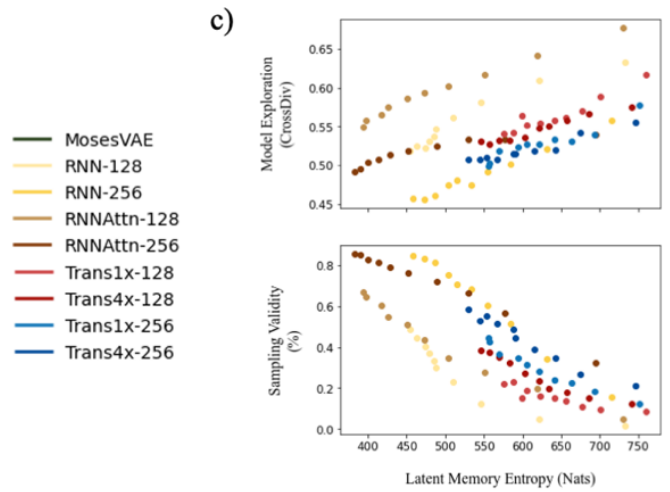

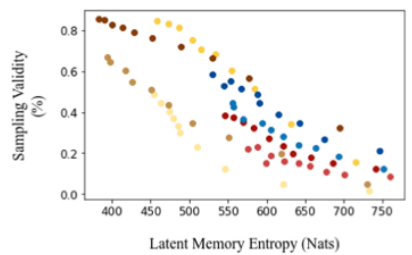

Figure 5. a) Visualizing a sample of 50 randomly selected molecular embeddings for three commonly observed memory structures (rows are a single molecular embedding and columns are the 128 latent dimensions). The information density (entropy) of each structure increases from left to right. b) Entropy of model memories during training (ZINC). Most models maintain the selective structure throughout training however the MosesVAE model undergoes a transition from selective to smeared at epoch 60. The KL Annealer prevents any model from experiencing posterior collapse. c) Exploration-validity tradeoff as a function of entropy when samples are drawn randomly from all latent dimensions. Cross diversity is evaluated only on valid molecules. The diversity of real molecular structures is shown to increase alongside model complexity as sampling validity decreases.

The progression of entropy during training is shown for each model type. We observe increases in the order MosesVAE $<$ RNNAttn $<$ RNN $<$ Transformer. The high entropy of the transformer models is expected and confirms that the molecular grammar they have learned is both complex and structurally meaningful. It is somewhat unexpected that the RNNAttn models have learned a less complex representation than even the simple recurrent models. Rather than learning grammatical rules, they have learned the most efficient way to distribute information to the bottleneck. The MosesVAE model has the most compressed representation, however it also has the worst reconstruction accuracy which can be attributed to the low information density and the selective to smeared transition at epoch 60 . We can now explore the relationship between complexity and the generative capabilities of the models, namely the validity of molecules sampled from the memory and their novelty when compared against the training set.

\section{Strategies for Exploring Chemical Phase Space}

A generative model is only as useful as its ability to generate interesting samples. Early molecular design VAEs struggled with generating valid molecules and research has placed a premium on improving the percent validity when 
a random sampling scheme is employed. However, we believe that exploration is undervalued in the current meta and that a slightly more error-prone model that prioritizes exploration may actually be more successful at discovering novel functional compounds. Novelty has previously been defined as the percentage of generated samples that are not present in the training set. ${ }^{19}$ We introduce another metric, cross diversity, which is defined as follows:

$$
\operatorname{CrossDiv}(\text { Gen, Train })=1-\frac{1}{\mid \text { Gen } \mid} \sum_{m_{\text {gen } \in \text { Gen }}} \max _{m_{\text {train } \in \text { Train }}} J\left(m_{\text {gen }}, m_{\text {train }}\right)
$$

where Gen and Train are the sample set and training set respectively, $m$ is a molecular fingerprint and $J\left(m_{1}, m_{2}\right)$ is the Jaccard similarity ${ }^{42}$ between two molecules. This metric will be close to 0 when all of the generated samples are very similar to molecules from the training set and close to 1 when they are all far from the training set. Therefore, it can be considered a measure of a model's tendency to explore new regions of phase space.

Table 2. Comparison of generative metrics for a low entropy (MosesVAE) and high entropy (Trans4x-128) model with random sampling

\begin{tabular}{cccccc} 
Model Type & Entropy (nats) & $\begin{array}{c}\text { \% Reconstruction } \\
\text { Accuracy (ZINC) }\end{array}$ & \% Validity & \% Novelty & Cross Diversity \\
\hline MosesVAE & 127.4 & 0.000 & 0.976 & 0.696 & 0.213 \\
Trans4x-128 & 546.4 & 0.998 & 0.365 & 0.998 & 0.530
\end{tabular}

The structure of a model's memory heavily influences its performance on these metrics. Random sampling favors the lowest entropy memories when the goal is to generate the highest proportion of valid molecules. However, low entropy models perform much worse on exploratory metrics (Table 2). In fact, this tradeoff between validity and exploration is a function of model entropy that is unavoidable and exists for all model types (Fig. 5c).

The difficulty in sampling from high entropy models is a result of the curse of dimensionality ${ }^{43}$ that appears within selective memory structures. High entropy dimensions contain all of the meaningful structural information within a model's memory (Fig. 6). When the memory is selectively structured, a high entropy means there are a greater number of meaningful dimensions and it becomes more difficult to avoid leaving "holes" where there is no mapping to a valid structure. This is not a problem for low entropy models as most of the dimensions are either meaningless or contain just a small amount of structural information. While we can easily sample from low entropy models, we miss out on the benefits of an information dense memory which is better at exploring chemical phase space.

Fortunately, while the diversity of generated molecules is mostly dependent on the complexity of the contextual relationships that have been embedded into the latent memory during training, validity can be optimized after training by considering sampling schemes other than random sampling. One potential strategy that requires no additional training and is trivial to implement is to target high entropy dimensions exclusively. This limits our search to the regions of chemical phase space which we know contain meaningful structural information. 


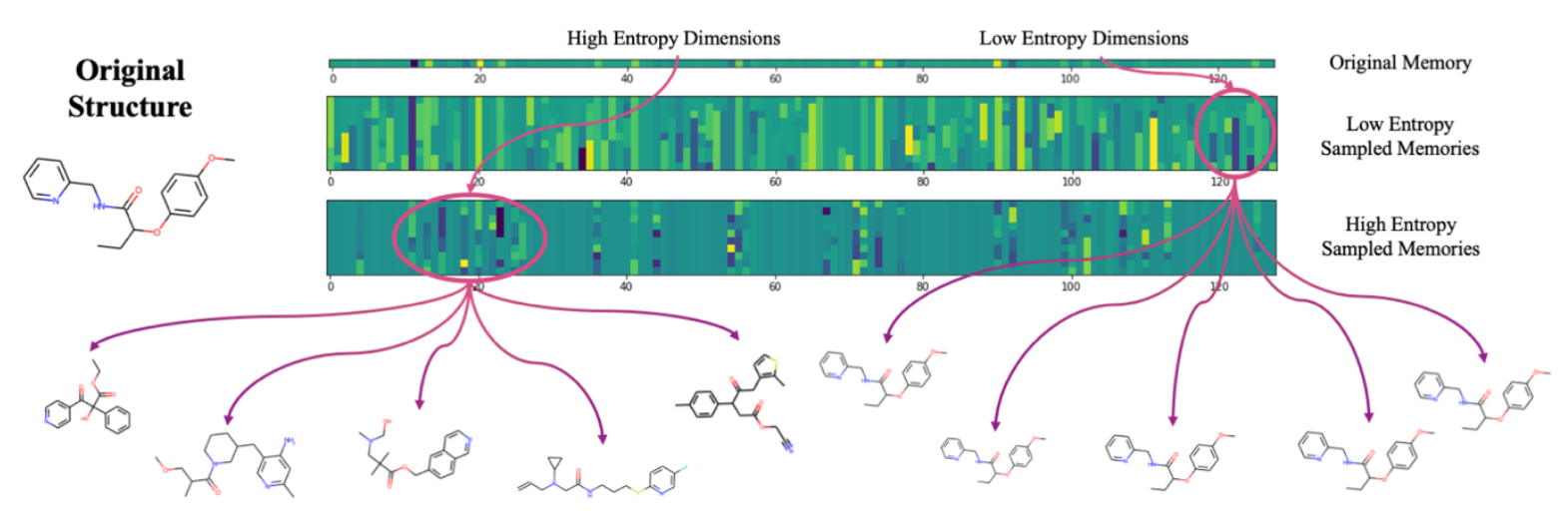

Figure 6. The result of exclusively sampling from low entropy dimensions (avg. entropy $<5$ nats) vs. high entropy dimensions. Sampling the low entropy dimensions has no effect on the decoded structure confirming that these dimensions are not used by the model. Sampling high entropy dimensions results in a diverse array of structures.

Fig. S9 shows validity and exploration for five different sampling schemes. By restricting the number of high entropy dimensions that are queried, we avoid the problems inherent to high-dimensional sampling and are able to increase the validity of generated molecules for all model types. This demonstrates the potential of exploiting novel sampling schemes that allow us to maintain the benefits of a complex, rich latent memory. For instance, we were able to achieve a $32.6 \%$ increase in the number of valid molecules generated by the Trans $4 x-256$ model, from 56.7 to $75.2 \%$ validity, while only reducing the cross diversity by $15.9 \%$, from 0.503 to 0.423 . Moreover, this range is still about two-times higher than the cross diversity of the MosesVAE. We also maintain the allure of the analytical and developmental possibilities that the highly interpretable transformer attention heads afford us by increasing the practical viability of these models in the short-term.

The choice of model type ultimately depends on the individual needs of the researcher, however we can submit a few broad recommendations. Smaller models tend to perform better on exploratory metrics whereas bigger models stick closer to the training set and generate a higher proportion of valid molecules. The addition of attention improves performance in both regards. Therefore, the RNNAttn-128 and RNNAttn-256 models are the most immediately practical. Transformers are the most interpretable and, in our view, have the highest potential for optimization and should be the focus of further development.

\section{Conclusions}

Herein, we have introduced the concept of attention to the field of molecular design, compared two novel architectures, RNNAttn and TransVAE, to the current state of the art and explored the downstream effect that the structure of the model memory has on a variety of sampling metrics. We find that transformers live up to their reputation based on their ability to learn complex substructural representations of molecular features, and we expect that there is an opportunity to expand our own chemical intuition as we continue to explore the relationships they have learned in more detail. The recurrent attention models, on the other hand, stand out for their superb practical performance exhibiting the best balance between reconstruction accuracy, sampling validity and cross diversity. 
We anticipate there will be two primary directions in which further research may proceed. The first is the direct application of attention based VAEs to real-world inverse design problems. There is a growing demand for biodegradable organic alternatives to toxic, high-value commodity chemicals in a number of different industries. ${ }^{44-46}$ Many of these involve molecules that are much larger than the average drug-like molecule and we are excited at the prospect of applying attention VAEs to these untapped areas. Generative algorithms have the potential to pair nicely with computational reaction networks such as $\mathrm{NetGen}^{47}$ and we can envision, as an example, a framework in which generated samples are used as the library for a high-throughput search of retrosynthetic pathways for the discovery of bioprivileged molecules. ${ }^{48}$

The second direction is the continued exploration and optimization of attention VAE architectures and their hyperparameters, particularly with regards to the formation of the latent memory during training. There is a definite potential for the implementation of more complex sampling schemes, for instance the two-stage $\mathrm{VAE}^{34}$ introduces a second model that takes the latent memory as an input and is better able to learn the true probability manifold of the input data. There is evidence that the use of a Gaussian prior restricts the model's ability to directly learn the true probability manifold and so it may be worth exploring alternatives like VampPrior as well. ${ }^{49}$

Perhaps the most worthwhile pursuit is to continue to develop our knowledge of how the model intuits and compresses structural information, as this could give us insight into novel objective functions that help us encourage the model to better shape its memory and relate it to other pieces of chemical information outside of the current scope. Although the field is advancing rapidly, we are still just at the threshold of the AI-dominated era that Marvin Minsky announced over a half century ago. ${ }^{50}$ There may be no aim more practical than furthering our own understanding of the nature of synthetic intelligence to push us further past that threshold. The latent conception of molecular structure is just one component within the broader field of organic chemistry and if coupled with a natural language model-based interpretation of scientific literature, high-throughput classical and quantum calculations, robotics driven lab-scale experimentation and an interactive environment in which our models can communicate and act upon their learning, we may finally begin to approach an intelligence that can solve problems at the pace we introduce them.

\section{Methods}

We tested three different model types - RNN, RNNAttn and Trans - for their ability to generate novel molecules. For each model type we also tested multiple architectures as summarized in Table 1. The Adam ${ }^{51}$ optimizer was used with an initial learning rate of $3 \mathrm{e}^{-4}$ and an annealer was used to linearly increase $\beta$ during training. We employed a scaling function that weighed the loss for each token based on its frequency of occurrence. All models were trained for 100 epochs unless stated otherwise. 
There are a couple of key differences between the MosesVAE and our own RNN implementation including the size and number of encoder/decoder layers, the use of bidirectionality for the encoder and the absence of batch normalization. For more details on the implementation of the MosesVAE please refer to Fig. S4/S5, Table 2 and the original paper by Polykovskiy et al. ${ }^{19}$ Further details about model construction and training can be found in the SI.

Two datasets were used to examine how the models perform on different training set distributions. The first is a modified version of the ZINC Clean Leads database ${ }^{52}$ with charged atoms removed and a molecular weight range of 250-350 Da. It contains a total of 1,936,963 molecules with an 80/10/10 train/test/dev split. The ZINC data was used to evaluate the models on a traditional AI-driven molecular design task - pharmaceutical discovery. The other is a

filtered subset of the PubChem compounds database. ${ }^{53}$ It contains molecules with a mean molecular weight of 348 $\mathrm{Da}$, a max of 2693.6 Da and includes some charged compounds with $\mathrm{N}^{+}$or $\mathrm{O}^{-}$containing moieties. Due to the size of the dataset after filtering, a subset of 5,000,000 molecules were randomly selected and used for training with an 80/10/10 train/test/dev split. The PubChem data was used to evaluate the models' performance on reconstructing molecules larger than those typically found in drug-like compound databases. The RDKit ${ }^{54}$ Python package was used for downstream analyses of generated molecules including SMILE validity, fingerprints, and physical property calculations.

\section{Supplementary Material}

See Supplementary Information for detailed descriptions of model architectures, hyperparameters and training decisions and all supplementary figures referenced in text. Saved model checkpoints and code to re-create all of the results reported in this article is available at https://github.com/oriondollar/TransVAE.

\section{Acknowledgements}

The authors would like to acknowledge David Juergens for his suggested edits to the final manuscript, Chowdhury Ashraf for his continued collaboration and work on optimizing the efficiency of parallel GPU computations. The NSF NRT program under award DGE-1633216 partially supported O.D. O.D., D.A.C.B., and J.P. acknowledge partial support for this research from NSF award OAC-1934292. This publication is also partially based upon work supported by the U.S. Department of Energy's Office Efficiency and Renewable Energy (EERE) under the Bioenergy Technologies Office Award Number DE-EE0008492. Computational resources for this work were provided by the Hyak supercomputer system of University of Washington 


\section{References}

(1) Kuhn, C.; Beratan, D. N. Inverse Strategies for Molecular Design; 1996.

(2) Sanchez-Lengeling, B.; Aspuru-Guzik, A. Inverse Molecular Design Using Machine Learning:Generative Models for Matter Engineering. Science. American Association for the Advancement of Science July 27, 2018, pp 360-365. https://doi.org/10.1126/science.aat2663.

(3) Polishchuk, P. G.; Madzhidov, T. I.; Varnek, A. Estimation of the Size of Drug-like Chemical Space Based on GDB-17 Data. Journal of Computer-Aided Molecular Design 2013, 27 (8), 675-679. https://doi.org/10.1007/s10822-013-9672-4.

(4) Elton, D. C.; Boukouvalas, Z.; Fuge, M. D.; Chung, P. W. Deep Learning for Molecular Generation and Optimization-a Review of the State of the Art. arXiv:1903.04388 [cs.LG] 2019.

(5) Beckner, W.; Ashraf, C.; Lee, J.; Beck, D. A. C.; Pfaendtner, J. Continuous Molecular Representations of Ionic Liquids. Journal of Physical Chemistry B 2020, 124 (38), 8347-8357. https://doi.org/10.1021/acs.jpcb.0c05938.

(6) Gaoy, W.; Coley, C. W. The Synthesizability of Molecules Proposed by Generative Models. J. Chem. Inf. Model 2020, 60, 5714-5723. https://doi.org/10.1021/acs.jcim.0c00174.

(7) Lim, J.; Ryu, S.; Kim, J. W.; Kim, W. Y. Molecular Generative Model Based on Conditional Variational Autoencoder for de Novo Molecular Design. Journal of Cheminformatics 2018, 10 (1), 31. https://doi.org/10.1186/s13321-018-0286-7.

(8) Kusner, M. J.; Paige, B.; Hernández-Lobato, J. M. Grammar Variational Autoencoder. 34th International Conference on Machine Learning, ICML 2017 2017, 4, 3072-3084.

(9) Liu, Q.; Allamanis, M.; Brockschmidt, M.; Gaunt, A. L. Constrained Graph Variational Autoencoders for Molecule Design. Advances in Neural Information Processing Systems 2018, 31, 7795-7804.

(10) Winter, R.; Montanari, F.; Noé, F.; Clevert, D. A. Learning Continuous and Data-Driven Molecular Descriptors by Translating Equivalent Chemical Representations. Chemical Science 2019, 10 (6), 1692-1701. https://doi.org/10.1039/c8sc04175j.

(11) Weininger, D. SMILES, a Chemical Language and Information System: 1: Introduction to Methodology and Encoding Rules. Journal of Chemical Information and Computer Sciences 1988, 28 (1), 31-36. https://doi.org/10.1021/ci00057a005.

(12) Krenn, M.; Häse, F.; AkshatKumar, N.; Friederich, P.; Aspuru-Guzik, A. Self-Referencing Embedded Strings (SELFIES): A 100\% Robust Molecular String Representation. Machine Learning: Science and Technology $2020,1$. Jin, W.; Barzilay, R.; Jaakkola, T. Junction Tree Variational Autoencoder for Molecular Graph Generation. arXiv:1802.04364 [cs.LG] 2018.

Gómez-Bombarelli, R.; Wei, J. N.; Duvenaud, D.; Hernández-Lobato, J. M.; Sánchez-Lengeling, B.; Sheberla, D.; Aguilera-Iparraguirre, J.; Hirzel, T. D.; Adams, R. P.; Aspuru-Guzik, A. Automatic Chemical Design Using a DataDriven Continuous Representation of Molecules. ACS Central Science 2018, 4 (2), 268-276. https://doi.org/10.1021/acscentsci.7b00572.

(15) Goyal, A.; Sordoni, A.; Maluuba, M.; Côté, M.-A.; Rosemary, N.; Mila, K.; Montréal, P.; Bengio, Y. Z-Forcing: Training Stochastic Recurrent Networks. arXiv:1711.05411 [stat.ML] 2017.

(16) Mohammadi, S.; O’dowd, B.; Paulitz-Erdmann, C.; Goerlitz, L. Penalized Variational Autoencoder for Molecular Design. ChemRxiv:7977131.v2 2019. https://doi.org/10.26434/chemrxiv.7977131.v2.

(17) Yan, C.; Wang, S.; Yang, J.; Xu, T.; Huang, J. Re-Balancing Variational Autoencoder Loss for Molecule Sequence Generation. arXiv. arXiv: New York, NY, USA October 1, 2019, pp 1-7. https://doi.org/10.1145/3388440.3412458. 
(18) Coley, C. W. Defining and Exploring Chemical Spaces. Trends in Chemistry. https://doi.org/10.1016/j.trechm.2020.11.004.

Polykovskiy, D.; Zhebrak, A.; Sanchez-Lengeling, B.; Golovanov, S.; Tatanov, O.; Belyaev, S.; Kurbanov, R.; Artamonov, A.; Aladinskiy, V.; Veselov, M.; Kadurin, A.; Johansson, S.; Chen, H.; Nikolenko, S.; Aspuru-Guzik, A.; Zhavoronkov, A. Molecular Sets (MOSES): A Benchmarking Platform for Molecular Generation Models. Frontiers in Pharmacology 2020, 11, 1931.

(20) Payne, J.; Srouji, M.; Yap, D. A.; Kosaraju, V. BERT Learns (and Teaches) Chemistry. arXiv:2007.16012 [q-bio.BM] 2020.

(21) Bahdanau, D.; Cho, K.; Bengio, Y. Neural Machine Translation by Jointly Learning to Align and Translate. arXiv:1409.0473 [cs.CL] 2014.

(22) Brown, T. B.; Mann, B.; Ryder, N.; Subbiah, M.; Kaplan, J.; Dhariwal, P.; Neelakantan, A.; Shyam, P.; Sastry, G.; Askell, A.; Agarwal, S.; Herbert-Voss, A.; Krueger, G.; Henighan, T.; Child, R.; Ramesh, A.; Ziegler, D. M.; Wu, J.; Winter, C.; Hesse, C.; Chen, M.; Sigler, E.; Litwin, M.; Gray, S.; Chess, B.; Clark, J.; Berner, C.; McCandlish, S.; Radford, A.; Sutskever, I.; Amodei, D. Language Models Are Few-Shot Learners. In 34th Conference on Neural Information Processing Systems (NeurIPS 2020), Vancouver, Canada; arXiv, 2020.

(23) Service, R. F. 'The Game Has Changed.' AI Triumphs at Protein Folding. Science 2020, 370 (6521), $1144-1145$. https://doi.org/10.1126/science.370.6521.1144.

(24) Anna Huang, C.-Z.; Vaswani, A.; Uszkoreit, J.; Shazeer, N.; Simon, I.; Hawthorne, C.; Dai, A. M.; Hoffman, M. D.; Dinculescu, M.; Eck, D. MUSIC TRANSFORMER: GENERATING MUSIC WITH LONG-TERM STRUCTURE. arXiv:1809.04281 [cs.LG] 2018.

(25) Elkins, K.; Chun, J. Can GPT-3 Pass a Writer's Turing Test? Journal of Cultural Analytics 2020, 17212. https://doi.org/10.22148/001c.17212.

(26) Floridi, L.; Chiriatti, M. GPT-3: Its Nature, Scope, Limits, and Consequences. Minds and Machines. Springer Science and Business Media B.V. November 1, 2020, pp 681-694. https://doi.org/10.1007/s11023-020-09548-1. Kingma, D. P.; Welling, M. Auto-Encoding Variational Bayes. In 2nd International Conference on Learning Representations, ICLR 2014 - Conference Track Proceedings; International Conference on Learning Representations, ICLR, 2014.

(28) Kullback, S.; Leibler, R. A. On Information and Sufficiency. The Annals of Mathematical Statistics 1951, 22 (1), $79-86$. https://doi.org/10.1214/aoms/1177729694.

(31) Burgess, C. P.; Higgins, I.; Pal, A.; Matthey, L.; Watters, N.; Desjardins, G.; Lerchner, A.; London, D. Understanding Disentangling in $\beta$-VAE. arXiv:1804.03599 [stat.ML] 2018.

(32) Blaschke, T.; Olivecrona, M.; Engkvist, O.; Bajorath, J.; Chen, H. Application of Generative Autoencoder in De Novo Molecular Design. Molecular Informatics 2018, 37 (1). https://doi.org/10.1002/minf.201700123.

(33) Vaswani, A.; Brain, G.; Shazeer, N.; Parmar, N.; Uszkoreit, J.; Jones, L.; Gomez, A. N.; Kaiser, Ł.; Polosukhin, I. Attention Is All You Need. In 31st Conference on Neural Information Processing Systems (NIPS 2017), Long Beach, CA, USA.; 2017; Vol. 30, pp 5998-6008.

(34) Dai, B.; Wipf, D. Diagnosing and Enhancing VAE Models. arXiv:1903.05789 [cs.LG] 2019. 
(35) Bahuleyan, H.; Mou, L.; Vechtomova, O.; Poupart, P. Variational Attention for Sequence-to-Sequence Models. In Proceedings of the 27th International Conference on Computational Linguistics; arXiv, 2018; pp 1672-1682.

(36) Liu, D.; Liu, G. A Transformer-Based Variational Autoencoder for Sentence Generation. In 2019 International Joint Conference on Neural Networks (IJCNN); Institute of Electrical and Electronics Engineers Inc., 2019; pp 1-7. https://doi.org/10.1109/IJCNN.2019.8852155.

(37) Lin, Z.; Winata, G. I.; Xu, P.; Liu, Z.; Fung, P. Variational Transformers for Diverse Response Generation. arXiv:2003.12738 [cs.CL] 2020.

(38) Wang, T.; Wan, X. T-CVAE: Transformer-Based Conditioned Variational Autoencoder for Story Completion. In Proceedings of the Twenty-Eighth International Joint Conference on Artificial Intelligence; 2019; pp 5233-5239.

(39) Sennrich, R.; Haddow, B.; Birch, A. Neural Machine Translation of Rare Words. arXiv: 1508.07909 2016.

(40) Shannon, C. E. A Mathematical Theory of Communication. The Bell System Technical Journal 1948, 27, 379-423.

(41) Batty, M.; Morphet, R.; Masucci, P.; Stanilov, K. Entropy, Complexity, and Spatial Information. Journal of Geographical Systems 2014, 16 (4), 363-385. https://doi.org/10.1007/s10109-014-0202-2.

(42) Jaccard, P. Nouvelles Recherches Sur La Distribution Florale. Bull. Soc. Vaud. Sci. Nat. 1908, 44, $223-270$.

(43) Bellman, R. Dynamic Programming. Science 1966, 153 (3731), 34-37. https://doi.org/10.1126/science.153.3731.34.

(44) Sheldon, R. A. The Road to Biorenewables: Carbohydrates to Commodity Chemicals. ACS Sustainable Chemistry and Engineering. American Chemical Society April 2, 2018, pp 4464-4480.

https://doi.org/10.1021/acssuschemeng.8b00376.

(45) Marzorati, S.; Verotta, L.; Trasatti, S. Green Corrosion Inhibitors from Natural Sources and Biomass Wastes. Molecules 2018, 24 (1), 48. https://doi.org/10.3390/molecules24010048.

(46) He, W.; Zhu, G.; Gao, Y.; Wu, H.; Fang, Z.; Guo, K. Green Plasticizers Derived from Epoxidized Soybean Oil for Poly (Vinyl Chloride): Continuous Synthesis and Evaluation in PVC Films. Chemical Engineering Journal 2020, 380, 122532. https://doi.org/10.1016/j.cej.2019.122532.

(47) Broadbelt, L. J.; Stark, S. M.; Klein, M. T. Computer Generated Pyrolysis Modeling: On-the-Fly Generation of Species, Reactions, and Rates. Ind. Eng. Chem. Res 1994, 33, 790-799.

(48) Shanks, B. H.; Keeling, P. L. Bioprivileged Molecules: Creating Value from Biomass. Green Chemistry 2017, 19 (14), 3177-3185. https://doi.org/10.1039/c7gc00296c.

(49) Tomczak, J. M.; Welling, M. VAE with a VampPrior. In International Conference on Artificial Intelligence and Statistics, AISTATS 2018; PMLR, 2018; pp 1214-1223.

(50) Minsky, M. Steps Toward Artificial Intelligence. Proceedings of the IRE. 1961, pp 8-30. https://doi.org/10.1109/JRPROC.1961.287775.

(51) Kingma, D. P.; Ba, J. L. Adam: A Method for Stochastic Optimization. In 3rd International Conference on Learning Representations, ICLR 2015 - Conference Track Proceedings; International Conference on Learning Representations, ICLR, 2015.

(52) Irwin, J. J.; Shoichet, B. K. ZINC - A Free Database of Commercially Available Compounds for Virtual Screening. Journal of chemical information and modeling 2005, 45 (1), 177. https://doi.org/10.1021/CI049714.

(53) Kim, S.; Thiessen, P. A.; Bolton, E. E.; Chen, J.; Fu, G.; Gindulyte, A.; Han, L.; He, J.; He, S.; Shoemaker, B. A.; Wang, J.; Yu, B.; Zhang, J.; Bryant, S. H. PubChem Substance and Compound Databases. Nucleic Acids Research 2016, 44 (D1), D1202-D1213. https://doi.org/10.1093/nar/gkv951.

(54) Landrum, G. RDKit: Open-Source Cheminformatics. RDKit: Open-source cheminformatics 2020, http://www.rdkit.org.

(55) Vaswani, A.; Bengio, S.; Brevdo, E.; Chollet, F.; Gomez, A. N.; Gouws, S.; Jones, L.; Kaiser, L.; Kalchbrenner, N.; Parmar, N.; Sepassi, R.; Shazeer, N.; Uszkoreit, J. Tensor2Tensor for Neural Machine Translation. In AMTA 2018 - 13th 
Conference of the Association for Machine Translation in the Americas, Proceedings; Association for Machine Translation in the Americas, 2018; Vol. 1, pp 193-199.

\section{TOC Image}

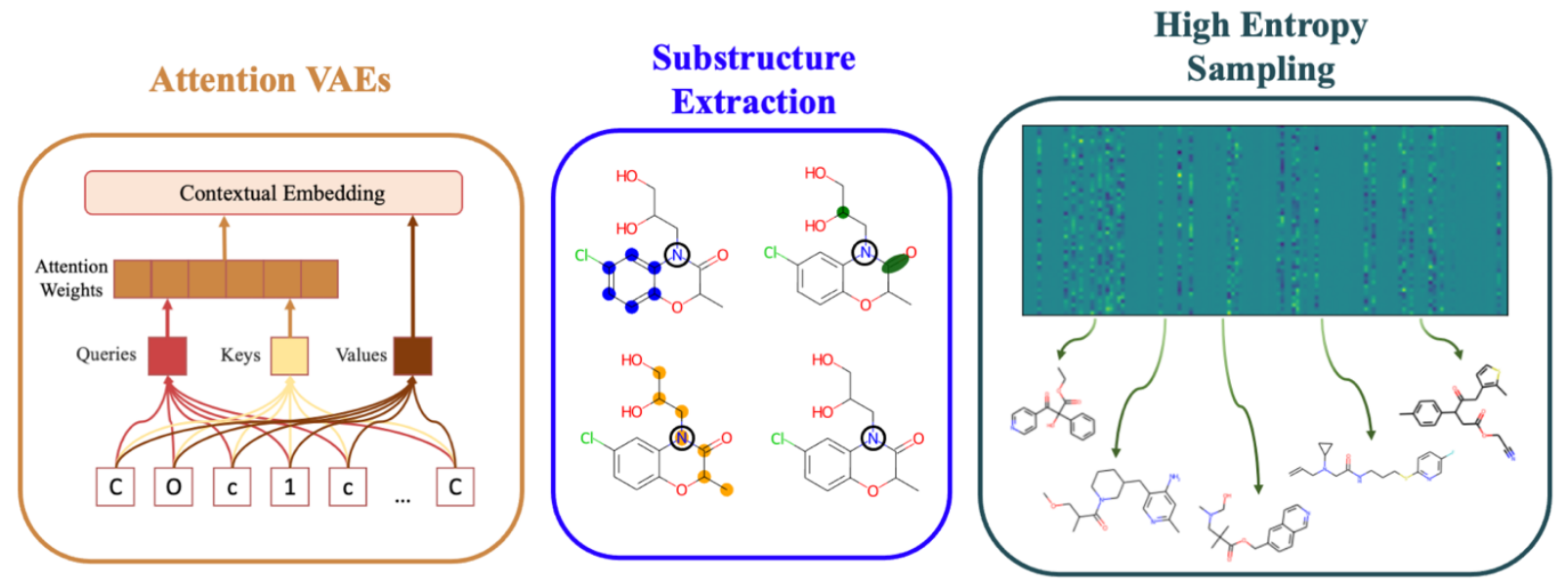

